\title{
Time-resolved linewidth measurements of a wavelength switched SG-DBR laser for optical packet switched networks
}

\author{
A.K. Mishra ${ }^{(1)}$, A.D. Ellis $^{(1)}$, L.P. Barry ${ }^{(2)}$, T. Farrell ${ }^{(3)}$ \\ (1) Photonic Systems Group, Department of Physics and Tyndall National Institute, University College Cork, Ireland \\ (2) School of Electronic Engineering, Dublin City University, Dublin 9, Ireland \\ (3) Intune Networks, 9 Beckett Way, Park West Business Park, Dublin 12, Ireland \\ e-mail:arvind.mishra@tyndall.ie
}

\begin{abstract}
We report time-resolved linewidth measurements of different sizes of optical packets under wavelength-switching for the first time and show that laser linewidth is significantly broadened during switching transition requiring $\sim 3$ s to attain its minimum value.

(C)2008 Optical Society of America

OCIS codes: (140.3600) Laser, tuneable; (130.4815) Optical switching devices; (060.4510) Optical communications
\end{abstract}

\section{Introduction}

The widely tunable sampled-grating distributed Bragg reflector (SG-DBR) lasers offer several opportunities to increase the capacity, functionality and flexibility of wavelength division multiplexed (WDM) networks. Considerable progress towards increasing the spectral efficiency of wavelength routed systems has been made [1]; however, beyond $0.8 \mathrm{~b} / \mathrm{s} / \mathrm{Hz}$, multi-level formats requiring coherent detection are likely to be required. In coherent communication systems using hard decision decoding, a linewidth between $0.2 \%-0.001 \%$ of bit rate is required at bit error rate (BER) of $10^{-9}$, depending on the configuration [2-3]. Forward error correction (FEC) allows a relaxation in this constraint by a factor of 3 [4]. In systems using wavelength switching, it is necessary that the laser stabilises sufficiently in a short time after the switching event. Whilst direct detection systems only require the laser frequency to stabilise, for coherent detection it is necessary for the phase noise to reach a sufficiently low level. Published results to date concentrate on the speed of wavelength tuning and the static linewidth [5-6], suggesting that coherent detection may be feasible with SG-DBR lasers.

In this letter, we report time-resolved linewidth measurements of a FPGA driven SG-DBR laser module under wavelength switching for the first time. Our method offers a spectral resolution of $5 \mathrm{MHz}$ and temporal resolution of 80ns, allowing us to determine the suitability of the laser for $40 \mathrm{Gsymbol} / \mathrm{s}$ coherent systems on a time scale comparable to the wavelength acquisition time. This study of wavelength switching characteristics is important for the application of such lasers in highly spectrally efficient coherent data transmission, and is also of interest for device design improvements.

\section{Experimental configuration}

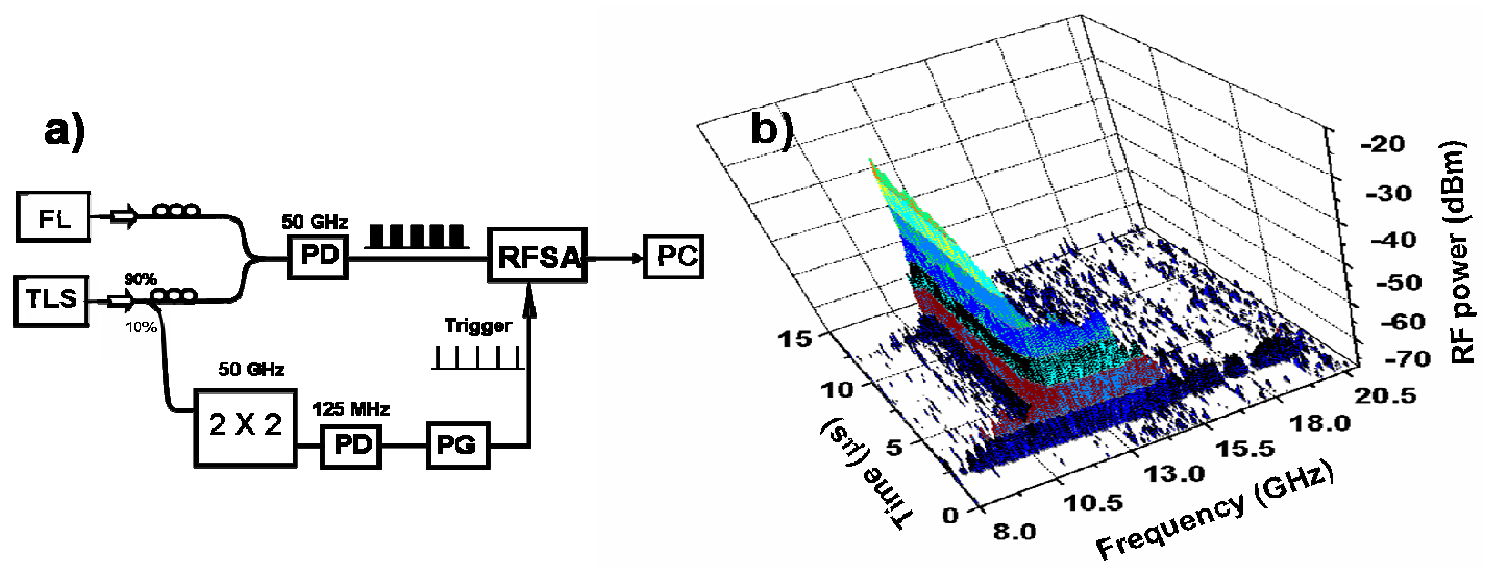

Figure 1a: Time resolved heterodyne linewidth measurement technique; TLS: tuneable laser source, FL: fibre laser, RFSA: radio frequency spectrum analyzer, PD: photodiode, PG: pulse generator, PC: personal computer; b) Full measured power spectrum 10 s packets of ch30 $(1553.45 \mathrm{~nm})$ following switching with $70 \mathrm{~ns}$ laser output blanking. 


\section{OTuC4.pdf}

The optical heterodyne technique for time resolved linewidth measurement of the SG-DBR laser is shown in figure 1a. A stable fibre laser with a narrow linewidth of $20 \mathrm{kHz}$ was used as a reference laser. The emission frequency of the fibre laser was tuned to $1553.36 \mathrm{~nm}, 11.76 \mathrm{GHz}$ away from ch30 (1553.45 nm) of the tunable SG-DBR laser. Two optical isolators were used to block optical feedback into the SG-DBR and reference laser and two polarization controllers were placed to maximize the interference signal by ensuring similar polarization states at the combining directional coupler. The optical output was sent to a $50 \mathrm{GHz}$ photodiode to measure the heterodyne beat signal on a $50 \mathrm{GHz}$ radio frequency spectrum analyzer (RFSA). The fitted linewidth of the ch30 output of the SG-DBR laser (Lorentzian lineshape) was found to be approximately $36 \mathrm{MHz}$ in static mode. Our fitting model provides a good fit over a dynamic range of $20 \mathrm{~dB}$ from the peak.

The tunable laser was switched between ch30 and various other channels with a variety of switching durations, and with a 70ns blanking period between each channel. $10 \%$ of output power of the SG-DBR was filtered by a $50 \mathrm{GHz}$ disinterlever and ch30 was thus detected with a low bandwidth photodiode to provide trigger synchronisation. The RFSA was triggered by this signal and set in zero span mode with a sweep time of $50 \mathrm{~s}$. The resolution bandwidth of the measurement was set to $1 \mathrm{MHz}$, giving a time resolution of $80 \mathrm{~ns}$. The centre frequency was tuned from 8 to 21 $\mathrm{GHz}$ in $5 \mathrm{MHz}$ steps and the resultant traces were transferred to a computer for processing using LabView. Combining these time traces, a time-dependent RF power spectrum of Ch30 following each switching event was obtained as shown in figure $2 \mathrm{a}$. The lineshape of the resultant optical power spectrum at any given moment after a switching event was obtained by slicing this 3D data set parallel to the frequency axis. Such time resolved spectra were curve fitted to a Lorentzian profile to determine the peak frequency and full-width at half-maximum (FWHM) linewidth. Note that during the rapid frequency deviations occurring immediately after the switching transient, the recorded linewidth would represent a convolution of the actual linewidth and the frequency change during the integration period (80ns). This gave a significant distortion of the lineshape up to 320ns after the switching event. To verify that the impact of this was small for the remainder of the data, we calculated the apparent linewidth due to the centre frequency drift by differentiating the frequency error measurements (shown in figure $3 b$ ).

\section{Results}

Figure 1(b) shows the measured time dependant RF power spectrum of $10 \mathrm{~s}$ ch30 packets after switching from channel 39 and blanking the laser output for $70 \mathrm{~ns}$ (to eliminate the spurious output from the TLS that occurs immediately after switching). In figure $2 \mathrm{a}$, we present a comparison of the frequency error measurements of ch30 taken using an optical method employing a $50 \mathrm{GHz}$ asymmetric MachZehnder disinterlever with the measurements derived from the RF spectrum analyser as described above (ch30 wavelength switching from ch39 and ch51). We found good agreement between both methods. Both the methods confirmed that the centre frequency of switching laser requires about $400 \mathrm{~ns}$ to reach a frequency error of $\pm 200 \mathrm{MHz}$, and reaches a stable operating point with a frequency drift error below $50 \mathrm{MHz}$ after between 400ns to $3 \mathrm{~s}$, depending on the switching channel combinations.
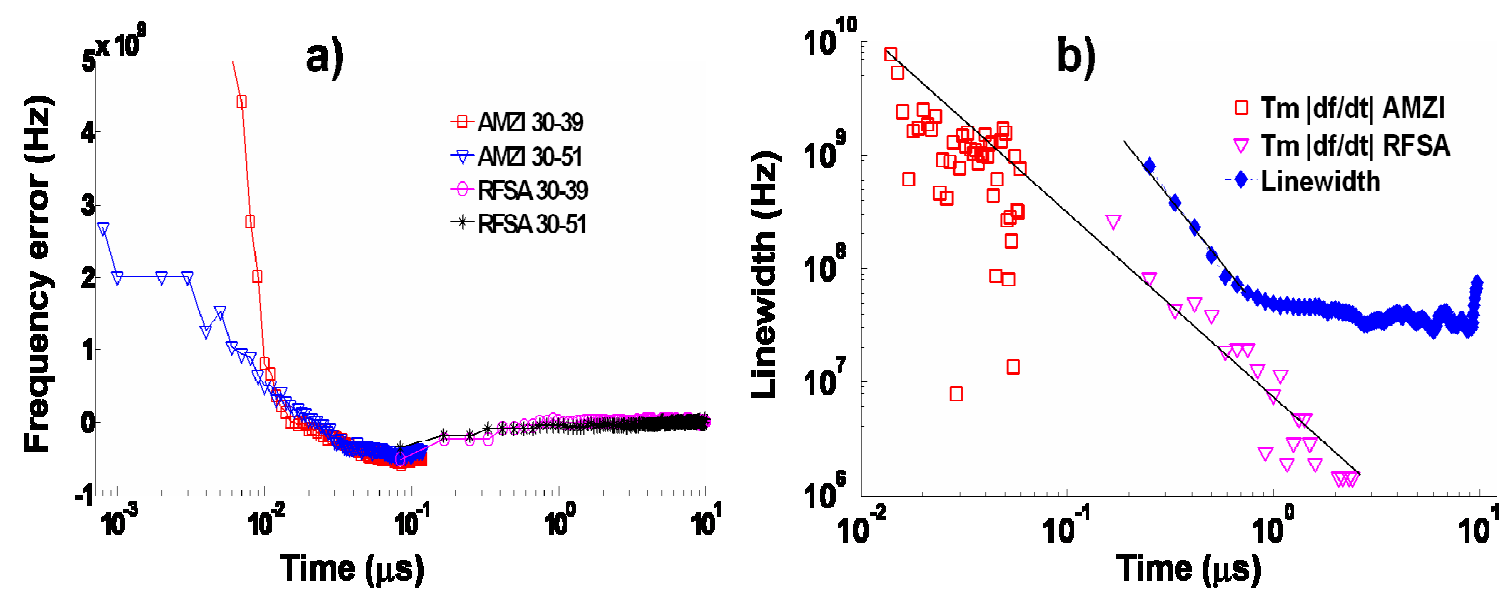

Figure 2a) Frequency error measurement using $50 \mathrm{GHz}$ disinterlever for 2.4 s packet and RF spectrum analyzer (RFSA) for 10 s packet of ch30 switching from ch39 and 51. b) A comparison of measured linewidth with the instantaneous frequency variation during the measurement interval versus time under wavelength switching from ch 39 to ch30. The curves are plotted versus time after wavelength switching

In figure $2 b$, we plot a typical measured linewidth and an estimate of the apparent linewidth that would be induced by centre frequency drift (by differentiating the frequency error measurements by both methods and integrating over 


\section{OTuC4.pdf}

the measurement interval). It is clear that the expected linewidth uncertainty from this effect is almost an order magnitude smaller than the actual measured linewidth and the result represent genuine linewidth broadening. The measured linewidth under various wavelength-switching combinations of ch 30, for $10 \mathrm{~s}$ packets are illustrated in figure 3(a). From this data, we observe that the linewidth falls to a level suitable for coherent detection after approximately $3 \mathrm{~s}$, corresponding to a residual wavelength drift rate of around $100 \mathrm{MHz} / \mathrm{s}$. We believe that the observed excess linewidth broadening is due to current fluctuations in the tuning sections of the laser which are controlled to draw the laser wavelength close to its target value. This is similar to the mechanisms which result in the broadened static linewidths of tuneable lasers [7], and our results (figure 2b) suggest that the linewidth broadening may be directly related to the rate of change of frequency.

In figure $3 b$, we present the time-resolved fitted linewidth results of ch30 for a number of different switching durations. It can be seen that the linewidth remains very high $(>200 \mathrm{MHz})$ for packet durations less than $1 \mathrm{~s}$ and slowly settles to its minimum of $36 \mathrm{MHz}$ (equal to the value in continuous mode) within 3 s for all longer packets. At the end of the each packet, we observed a marked increase in the linewidth up to $200 \mathrm{MHz}$ for the longest packets which is not associated with a corresponding change in the laser frequency. We believe that this is due to a slight pickup of noise from the drive electronics as it configures for the next wavelength switch.
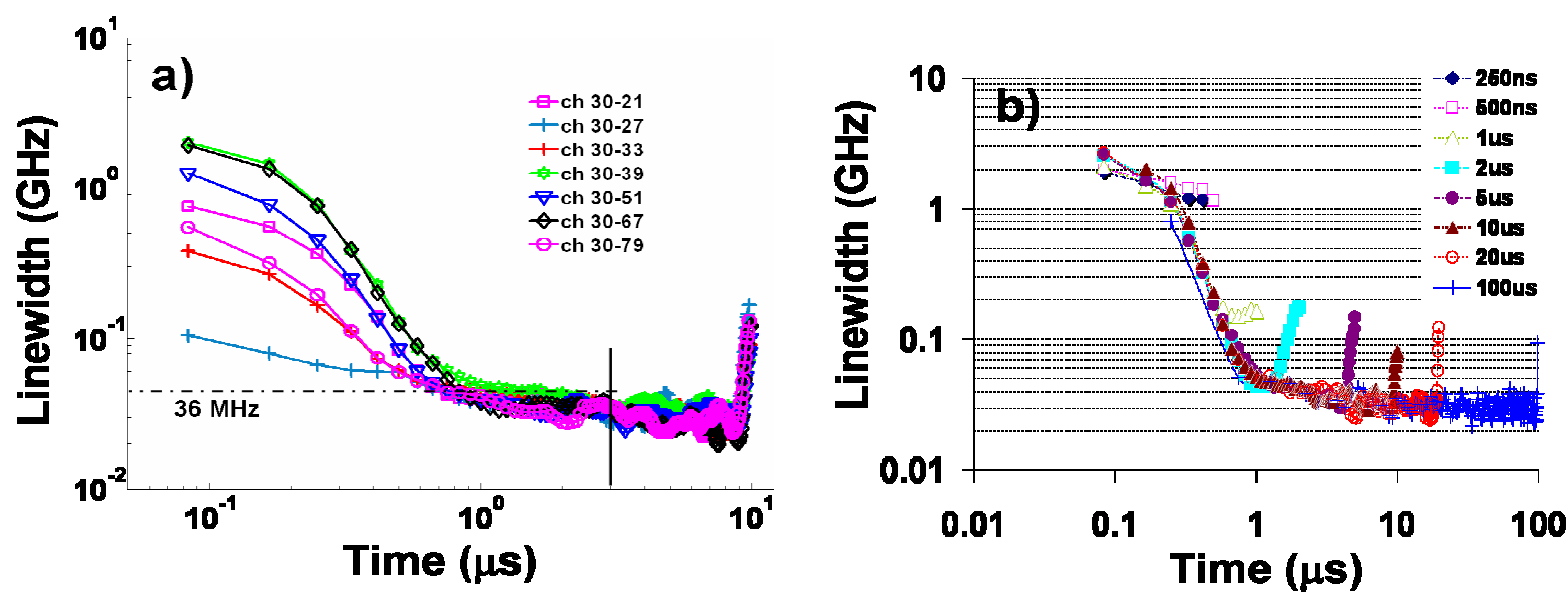

Figure 3a Plot of dynamic linewidth of ch30 of 10 s packet for various channel switching combinations. b) Measured time resolved linewidth versus time for different packet lengths. The curves are plotted versus time after wavelength switching

\section{Discussion and Conclusions}

We have investigated the time resolved linewidth characteristics of a SG-DBR laser under wavelength switching for the first time to our knowledge. Our experimental results showed that tuneable laser linewidth is significantly broadened during switching, requiring $\sim 3$ s to attain its minimum value, which corresponds to the laser emission wavelength reaching its steady state value. This behaviour and its relationship to transient time constants within a particular laser structure, particularly thermalisation characteristics should be taken into account when designing transmission systems and tuneable laser modules for coherent applications. The measured device appears suitable for coherent detection applications in burst switched networks with burst lengths significantly longer than 3 s, and provisioning applications in circuit switched networks.

This work is supported by Science Foundation Ireland under grants 06/IN/I969 and 03/IN3/I560. The authors would like to acknowledge R. Web, R.J. Manning, and D. Cotter for useful discussions.

\section{References}

[1] M.Duelk et al., "Fast Packet Routing in a 2.5 Tb/s Optical Switch Fabric with 40 Gb/s Duobinary Signals at 0.8 b/s/Hz Spectral Efficiency", Proc OFC'03, Atlanta, paper PD8-1, 2003.

[2] L.G.Kazovsky, et al.,"Homodyne Phase-Shift-Keying Systems: Past Challenges and Future Opportunities", JLT, 24(12), 2006, pp. 4876-4883. [3] David W. Smith, "Techniques for Multigigabit Coherent Optical Transmission”, J. Lightwave Technology, LT-5(10), 1987 pp. $1466-1478$.

[4] I.Garrett and G.Jacobsen, "The Effect of Laser Linewidth on Coherent Optical Receivers with Nonsynchronous Demodulation", JLT, LT-5(4), 1987, pp. 551-560.

[5] Y.A.Akulova, et al., "Widely Tunable Electroabsorption-Modulated Sampled-Grating DBR Laser Transmitter", IEEE JSTQE, 8(6), 2002, pp. 1349-1357.

[6] J. E.Simsarian,et al., "Less Than 5-ns Wavelength Switching With an SG-DBR Laser", IEEE Photon. Technol. Lett., 18(4), 2006 pp.565-567. [7] M-C. Amann and R. Schimpe, "Excess linewidth broadening in wavelength-tunable laser diodes," Electron. Lett., 26(5), 1990, pp.279-280. 